\title{
Large genomic rearrangements in NIPBL are infrequent in Cornelia de Lange Syndrome
}

\author{
Zahurul A Bhuiyan*, ${ }^{*}$, Helen Stewart ${ }^{2}$, Egbert J Redeker ${ }^{1}$, Marcel MAM Mannens ${ }^{1}$ \\ and Raoul CM Hennekam ${ }^{3,4}$
}

\begin{abstract}
${ }^{1}$ Department of Clinical Genetics, Academic Medical Centre, University of Amsterdam, Amsterdam, The Netherlands; ${ }^{2}$ Department of Clinical Genetics, Churchill Hospital, Headington, Oxford, UK; ${ }^{3}$ Clinical and Molecular Genetics Unit, Institute of Child Health, Great Ormond Street Hospital for Children, UCL, London, UK; ${ }^{4}$ Department of Paediatrics, Academic Medical Centre, University of Amsterdam, Amsterdam, The Netherlands
\end{abstract}

Cornelia de Lange Syndrome (CdLS) is a multiple congenital anomaly syndrome characterized by a distinctive facial appearance, malformations of the upper limbs, and delay in growth and development. Mutations in NIPBL are associated with CdLS in $27-56 \%$ of cases and have been reported as point mutations, small insertions and deletions in coding regions, regulatory regions and at splice junctions. All previous studies used PCR-based exon-scanning methodologies that do not allow detection of large genomic rearrangements. We studied the relative copy number of NIPBL exons in a series of $50 \mathrm{CdLS}$ probands, negative for NIPBL mutations, by multiplex ligation-dependent probe amplification (MLPA). In a single patient, we found a $5.2 \mathrm{~kb}$ deletion encompassing exons 41-42 of NIPBL. Our studies indicate that large NIPBL rearrangements do occur in CdLS but are likely to be infrequent events.

European Journal of Human Genetics (2007) 15, 505-508. doi:10.1038/sj.ejhg.5201776; published online 31 January 2007

Keywords: Cornelia de Lange Syndrome; NIPBL; mutation; gene deletion; MLPA

\section{Introduction}

Cornelia de Lange syndrome (CdLS; MIMno. 122470) is a multiple congenital anomaly syndrome characterized by a distinctive facial appearance, prenatal and postnatal growth deficiency, malformations of the upper extremities, psychomotor delay and behavioural problems. ${ }^{1}$ In 2004 , two groups reported that NIPBL gene mutations can be responsible for CdLS. ${ }^{2,3}$ NIPBL is located on chromosome $5 \mathrm{p} 13$, and is the human homologue of the Drosophila Nipped-B gene, which belongs to the family of chromosomal adherins involved in chromatid cohesion processes and enhancer-promoter communication in Drosophila. ${ }^{4-5}$

*Correspondence: Dr ZA Bhuiyan, Department of Clinical Genetics, Academic Medical Centre, University of Amsterdam, Meibergdreef 15, 1105 AZ, Amsterdam, The Netherlands.

Tel: + 3120 6009025; Fax: + 3120 5669389;

E-mail: Z.A.Bhuiyan@amc.uva.nl

Received 27 September 2006; revised 27 November 2006; accepted 6 December 2006; published online 31 January 2007
The exact function of the human NIPBL gene product, called delangin, is unknown. Recently an X-linked form of CdLS was postulated, caused by mutations in SMC1L1. ${ }^{6}$ The clinical features seemed less expressed, especially regarding growth, development and limbs, but more patients are needed to allow for firm conclusions in this.

To date, NIPBL mutations have been identified in $27-56 \%$ (mean $42 \%$ ) of CdLS cases. ${ }^{2-3,7-12}$ The studies used PCR-based exon-scanning methodologies such as single-stranded conformation polymorphism (SSCP) analysis, denaturing high-performance liquid chromatography (DHPLC) analysis, and direct sequencing of PCR-amplified coding regions for mutation screening. Such studies can only identify point mutations or small insertions and deletions in coding regions or at splice junctions but do not detect large duplications or deletions.

To determine whether large genomic rearrangements are frequently responsible for CdLS in the group of patients that are mutation negative with PCR based exon-scanning 
techniques, we analyzed a series of mutation-negative CdLS probands for large genomic rearrangements in $N I P B L$ by multiplex ligation-dependent probe amplification (MLPA) analysis, ${ }^{13}$ which uses a quantitative multiplex approach for determining the relative copy number of gene exons.

\section{Patients and methods Patients}

The patient group consisted of 50 patients ( 22 boys, 28 girls; age 15-months to 46-years) with CdLS in whom we could not detect a NIPBL mutation in coding region or splice site in the NIPBL gene by DHPLC-DNA sequencing, or direct sequencing using primers in flanking intronic sequences. ${ }^{7}$ Among them 17 patients formed part of an earlier study, ${ }^{7}$ the other 33 patients are unreported previously. The study was performed according to a protocol approved by the local ethics committee. Informed consent was obtained from the parents of all patients.

\section{MLPA analysis}

Probes for MLPA analysis of NIPBL exons 1-47 (SALSA MLPA Kit P141 and P142) was purchased from MRC Holland (Amsterdam, The Netherlands). MLPA was performed according to the manufacturer's instructions. In brief, 500 ng DNA was denatured and hybridized overnight at $60^{\circ} \mathrm{C}$ with SALSA probe mix. After treating the samples with ligase 65 for $15 \mathrm{~min}$ at $54^{\circ} \mathrm{C}$, PCR amplification was performed with the specific SALSA FAM PCR primers. Electrophoresis of PCR products was performed using an ABI PRISM 310. Data analysis was performed by exporting the peak areas to a Microsoft Excel file. Sample-related and peak-related differences in PCR and electrophoresis efficiency were corrected by first calculating the peak area relative to the sum of peak areas per sample and subsequently expressing each normalized peak area relative to the mean of that peak across samples. To detect deviating peaks, each normalized peak was divided by the mean of that peak in the control samples. Peak heights outside the range 0.7-1.3 times the control peak height were considered abnormal, with those below 0.7 representing deletions and those above 1.3 representing duplications. Several control samples were included in each MLPA test. Each result was confirmed by two independent experiments.

\section{Confirmation and analysis of the large deletion in NIPBL by PCR}

For confirmation of the deletion in NIPBL and to estimate the size of the deleted fragment and locate its boundaries, PCR was performed with the following primer combination: 5'-CCAGTCAAGTGTACGCCACTTTGCCCTAA-3' (forward), located in exon 40 and $5^{\prime}$-ACAACGGCTCTTCCTGT GTCTGGTATGGA-GTCTGGTATGGA-3' (reverse), located in exon 43. Sequence primers used for precise deletion mapping were: 5'-AGTGATACGATGAATTCCT-3', 5'-CACT CACTGCCACCTTGA-3' ${ }^{\prime}$, 5'-TGCTATCAGTTTCACACTG-3, 5'-AGCAGAGTCTATACCCTTC-3'.

\section{Results}

Detection of a large deletion in NIPBL

MLPA yielded normal results in 49 probands. An aberrant exon copy number was detected in one proband. In the latter patient, after quantification, compared to all other exons copy number of exons 41 and 42 seems to be halved (Figure 1a and b) suggesting that exons 41 and 42 of one allele was deleted. PCR amplification using the exons 40 and 43 specific primers yielded a 2.6-kb fragment, which confirmed this finding and revealed that the region deleted was approximately $5.2 \mathrm{~kb}$ (Figure 1c). PCR product yielded a 7.8-kb fragment with DNA from control individuals (Figure 1c). DNA sequencing of the $2.6-\mathrm{kb}$ fragment from the patient revealed that the heterozygous deletion consisted of deletion of 1095 bp of intron 40, exon 41, intron 41, exon 42 and partial deletion of intron 42 (c.6955-1095_c.7263+3344del5227). No such deletion was detected in parental samples.

\section{Phenotype of CdLS patient with large NIPBL deletion} The girl is the third child of healthy, second cousin parents of Pakistani extraction. The pregnancy was complicated by episodes of preterm labour and her mother received treatment with dexamethasone on three occasions. She was born at 36 weeks and 5 days weighing $2.4 \mathrm{~kg}$ (9th centile) and with a head circumference of $31 \mathrm{~cm}$ (9th centile). APGAR scores were 5 and 9 after 1 and $5 \mathrm{~min}$, respectively, and she required short-term facial oxygen, was hypoglycaemic, fed poorly and had apnoeas. She had general hirsutism, was brachycephalic, a synophrys, neatly arched eyebrows, depressed nasal bridge with anteverted nares, smooth philtrum with a central raphe, a thin upper lip and posteriorly rotated ears. Parents did not give permission to publish a picture of their child. Her hands were small, with short tapering digits, single palmar creases bilaterally and left fifth finger clinodactyly, her feet were also small with unilateral metatarsus adductus. Echocardiography demonstrated tetralogy of Fallot for which she underwent a successful Blalock-Taussig shunt procedure. At 18 months of age she weighed $7 \mathrm{~kg}(\ll 0.4$ th centile). Further shunt surgery was required as her left anterior descending artery originated from the right coronary artery and crossed the right ventricular outflow tract. A renal ultrasound showed a small scarred right kidney, left pelvic dilation with loss of corticomedullary differentiation. She had severe feeding problems, reflux (treated by fundoplication) and was hypertonic. At 21 months of age she was severly developmentally delayed, sitting with support, reaching, laughing and babbling, with 
a

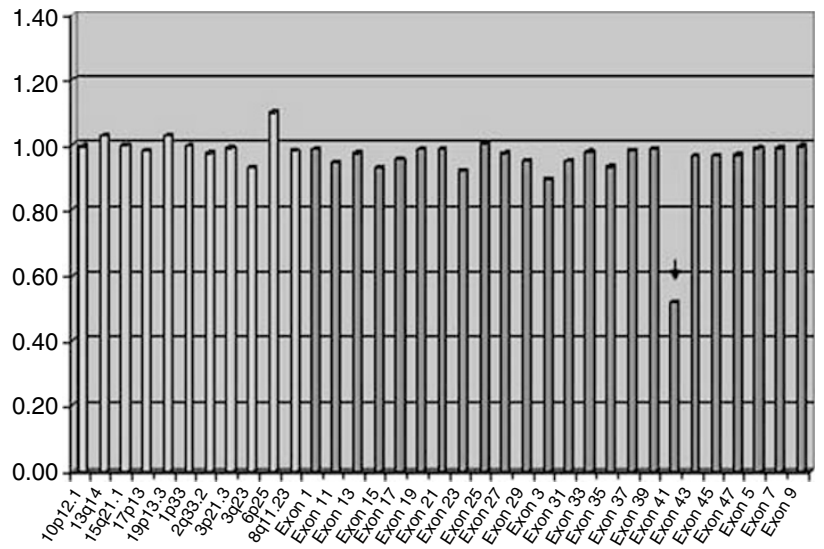

b

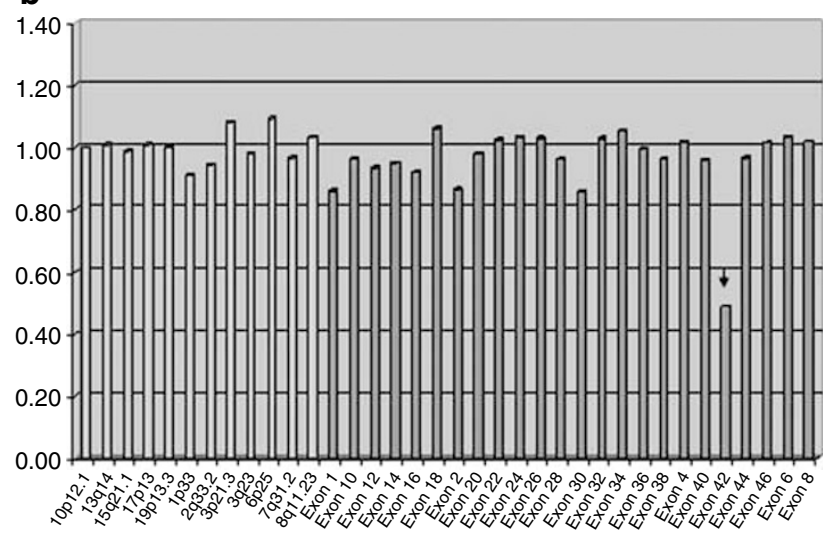

C

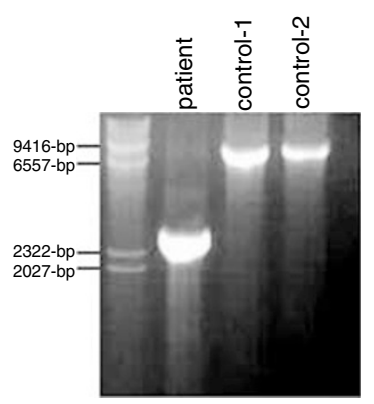

Figure 1 MLPA results. ( $\mathbf{a}$ and $\mathbf{b}$ ). The height of the columns represents the dosage of the respective segments in the genomic DNA with two alleles (value of about one corresponds to two alleles). The two-allele dosage for NIPBL exons 41 and 42 (arrow marked) was found in the range of 0.5 . The control fragments (left 11-columns) in both panels are near the value of about 1 which corresponds to two alleles. (c) Long range PCR from exons 40-43 confirmed the deletion and revealed that the region deleted was $5.2 \mathrm{~kb}$. A PCR product of 2.6$\mathrm{kb}$ was obtained with DNA from the affected girl and 7.8-kb fragment with DNA from two controls. Owing to preferential amplification of the deleted allele, the larger normal allele was not amplified in the patient. Therefore, the normal $7.8-\mathrm{kb}$ fragment is absent in the patient lane. normal hearing and vision. She was hypertonic and had seizures from the age of 3.5 years. A CT scan showed slight atrophy of the pons and medulla but no other intracerebral abnormality.

\section{Discussion}

Using MLPA analysis, we identified a $5.2 \mathrm{~kb}$ deletion in NIPBL in a girl with an expressed CdLS phenotype (classical facial features as defined elsewhere; ${ }^{14}$ expressed postnatal growth retardation; severely impaired cognitive functioning) predominantly found in patients with truncating mutations. ${ }^{7,12}$ The $5.2 \mathrm{~kb}$ deletion is expected to cause premature termination of translation of NIPBL leading to a non-functional or severely debilitated protein; therefore, disruption of exons 41 and 42 is expected to have serious consequences on the protein structure. The truncation would be likely to abolish the recently discovered serine phosphorylation sites in the C-terminal region that are important for mitotic spindle formation. ${ }^{15}$ To define more precisely the $5^{\prime}$ and $3^{\prime}$ boundaries of the deletion, PCR amplified fragments were analysed by sequencing. We found two Alu repeats, one 185-bp upstream to the break junction, followed by a second Alu repeat located 327-bp downstream to the first Alu element. This second Alu repeat was also deleted in the patient, meaning that this deletion did not occur owing to an Alu mediated rearrangement as described in chromosome rearrangements. ${ }^{16,17}$

We found one large deletion in 50 CdLS patients in whom previous investigations failed to show a mutation. Until now no large deletions have been identified in the NIPBL gene by FISH analysis, however, FISH lacks the resolution of MLPA in detecting smaller deletions. No other studies using MLPA have been reported. Large intragenic deletions, such as the one described here, are likely to be infrequent events, but screening using MLPA is still useful in CdLS patients negative in routine mutation screening strategies. Recently a mutation in the 5 'non-coding region of NIPBL was reported in one of 21 studied CdLS patients, ${ }^{9}$ but no mutation in the NIPBL promoter was found by partial analysis in 11 other patients. ${ }^{11}$ In addition, SMC1L1 mutations will explain the occurrence of CdLS in a certain percentage of patients, ${ }^{6}$ although no reliable figures are available at present. It remains well possible that other, as yet unknown genes, for instance other genes involved in chromatid cohesion processes, will be found to be responsible for the group of CdLS patients that remain mutation-negative at present.

\section{Acknowledgements}

We thank all patients and their parents, and all referring physicians for their cooperation in this study. 


\section{References}

1 Gorlin RJ, Cohen MM, Hennekam RC: Syndromes of the Head and Neck (4th edn.). Oxford Medical Press: New York, 2001, pp $372-377$.

2 Krantz ID, McCallum J, DeScipio C et al: Cornelia de Lange syndrome is caused by mutations in NIPBL, the human homolog of Drosophila melanogaster Nipped-B. Nat Genet 2004; 36: 631-635.

3 Tonkin ET, Wang TJ, Lisgo S, Bamshad MJ, Strachan T: NIPBL, encoding a homolog of fungal Scc2-type sister chromatid cohesion proteins and fly Nipped-B, is mutated in Cornelia de Lange syndrome. Nat Genet 2004; 36: 636-641.

4 Rollins RA, Morcillo P, Dorsett D: Nipped-B, a Drosophila homologue of chromosomal adherins, participates in activation by remote enhancers in the cut and Ultrabithorax genes. Genetics 1999; 152: $577-593$.

5 Rollins RA, Korom M, Aulner N, Martens A, Dorsett D: Drosophila nipped-B protein supports sister chromatid cohesion and opposes the stromalin/Scc3 cohesion factor to facilitate long-range activation of the cut gene. Mol Cell Biol 2004; 24: 3100-3111.

6 Musio A, Selicorni A, Focarelli ML et al: X-linked Cornelia de Lange syndrome owing to SMC1L1 mutations. Nat Genet 2006; 38: $528-530$.

7 Bhuiyan ZA, Klein M, Hammond P et al: Genotype-phenotype correlations of 39 patients with Cornelia de Lange syndrome: the Dutch experience. J Med Genet 2006; 43: 568-575.

8 Bhuiyan ZA, Zilfalil BA, Hennekam RC: A Malay boy with the Cornelia de Lange syndrome: clinical and molecular findings. Singapore Med J 2006; 47: 724-727.

9 Borck G, Zarhrate M, Cluzeau C et al: Father-to-daughter transmission of Cornelia de Lange syndrome caused by a mutation in the $5^{\prime}$ untranslated region of the NIPBL gene. Hum Mutat 2006; 27: 731-735.

10 Gillis LA, McCallum J, Kaur M et al: NIPBL mutational analysis in 120 individuals with Cornelia de Lange syndrome and evaluation of genotype-phenotype correlations. Am J Hum Genet 2004; 75: $610-623$.

11 Miyake N, Visser R, Kinoshita A et al: Four novel NIPBL mutations in Japanese patients with Cornelia de Lange syndrome. Am J Med Genet 2005; 135: 103-105.

12 Yan J, Saifi GM, Wierzba TH et al: Mutational and genotype-phenotype correlation analyses in 28 Polish patients with Cornelia de Lange syndrome. Am J Med Genet 2006; 140: $531-1541$.

13 Schouten JP, McElgunn CJ, Waaijer R, Zwijnenburg D, Diepvens F, Pals G: Relative quantification of 40 nucleic acid sequences by multiplex ligation-dependent probe amplification. Nucleic Acids Res 2002; 30: e57.

14 Allanson JE, Hennekam RCM, Ireland M: De Lange syndrome: subjective and objective comparison of the classical and mild phenotypes. J Med Genet 1997; 34: 645-650.

15 Nousiainen M, Sillje HH, Sauer G, Nigg EA, Korner R: Phosphoproteome analysis of the human mitotic spindle. Proc Natl Acad Sci USA 2006; 103: 5391-5396.

16 Myerowitz R, Hogikyan ND: A deletion involving Alu sequences in the beta-hexosaminidase alpha-chain gene of French Canadians with Tay-Sachs disease. J Biol Chem 1987; 262: 15396-15399.

17 Matejas V, Huehne K, Thiel C, Sommer C, Jakubiczka S, Rautenstrauss B: Identification of Alu elements mediating a partial PMP22 deletion. Neurogenetics 2006; 7: 119-126. 